\title{
miR-345 inhibits tumor metastasis and EMT by targeting IRF1-mediated mTOR/STAT3/AKT pathway in hepatocellular carcinoma
}

\author{
MIAO YU, HUANZHOU XUE, YADONG WANG, QUAN SHEN, QINGFENG JIANG, \\ XIAO ZHANG, KE LI, MENG JIA, JIANGKUN JIA, JIAN XU and YUWEI TIAN
}

Department of Hepatobiliary Surgery, People's Hospital of Zhengzhou University, Zhengzhou, Henan 450003, P.R. China

Received November 16, 2016; Accepted January 9, 2017

DOI: $10.3892 /$ ijo.2017.3852

\begin{abstract}
MicroRNAs (miRNAs) have been reported to play critical roles in tumor progression including hepatocellular carcinoma (HCC). Thus, the underlying mechanisms need further investigation. Previous study reported that loss of miR-345 expression indicated a poor prognosis of HCC patients. This study evaluated whether loss of miR-345 could promote the tumor metastasis and epithelial-mesenchymaltransition (EMT) of HCC by targeting interferon regulatory factor 1 (IRF1)-mediated mTOR/STAT3/AKT signaling. Underexpression of miR-345 was identified in 65 cases of human HCC compared to matched tumor-adjacent tissues by qRT-PCR. Moreover, we found that reduced expression of mi-345 was observed in HCC cell lines. The restoration of miR-345 inhibited cell migration and invasion in HCCLM3 cells, while its loss facilitated the cell mobility of HepG2 cells. Furthermore, miR-345 overexpression reduced lung metastases of HCC cells in nude mice. Notably, miR-345 overexpression prohibited, while its knockdown enhanced the EMT process of HCC cell lines in vitro. Bioinformatics software predicted that IRF1 was a direct target of miR-345. We then observed the negative regulation of miR-345 on IRF1 protein expression and the direct binding between them was further verified by dual-luciferase assays in HCC cells. In addition, overexpression of IRF1 mRNA was inversely correlated with the level of miR-345 in HCC specimens. Restoration of IRF1 resulted in promoted EMT and cell mobility in miR-345 overexpressing HCCLM3 cells. It was found that mTOR/ STAT3/AKT pathway and its downstream targets including Slug, Snail and Twist may be involved in IRF1 mediated EMT process. In conclusion, miR-345 acts as an inhibitor of
\end{abstract}

Correspondence to: Dr Huanzhou Xue, Department of Hepatobiliary Surgery, People's Hospital of Zhengzhou University, 7 Weiwu Road, Zhengzhou, Henan 450003, P.R. China

E-mail: xhz_zzu@163.com

Key words: miR-345, hepatocellular carcinoma, metastasis, signaling pathway, epithelial-mesenchymal-transition, interferon regulatory factor 1
EMT process in HCC cells by targeting IRF1 and this study highlights the potential effects of miR-345 on prognosis and treatment of HCC.

\section{Introduction}

Hepatocellular carcinoma (HCC), the most frequent primary liver cancer, and the third leading cause of cancer-related death in the world (1). Although the treatments have improved in recent years, metastasis and recurrence of HCC compromise the efficiency of new therapies, and the survival of HCC patients remain dismal (2). Better understanding of the metastasis mechanism is required.

MicroRNAs (miRNAs), small non-coding RNAs, posttranscriptionally regulate protein expression levels via directly binding to mRNAs of potentially hundreds of genes at the 3'-untranslated regions (3'-UTR) (3). Dysregulated miRNAs contribute to cancer initiation and progression by acting as proto-oncogenes or tumor suppressor genes (4). miR-345 were shown highly expressed in malignant mesothelioma compared with normal samples (5). Furthermore, miR-345 expression was increased and might play a critical role in malignant transformation of oral carcinoma (6). miR-345 was implicated in cisplatin resistance of MCF-7 by targeting multidrug resistance-associated protein 1 (MRP1) (7). In colorectal cancer, underexpression of miR-345 conferred to lymph node metastasis and worse histological type, and its restoration inhibited cancer cell proliferation and invasion $(8,9)$. Moreover, miR-345 expression was downregulated in non-small cell lung cancers (NSCLC) and its low expression was correlated with malignant clinical parameters and poor prognosis (10). Loss of miR-345 was reported to confer apoptosis resistance to pancreatic cancer (PC) cells (11), and miR-345 inhibited prostate cancer cell proliferation and mobility by suppressing Smad1 (12). Rare studies reported the correlation between miR-345 and HCC. Shiu et al reported that hepatitis C virus (HCV) core protein upregulated the expression of miR-345, which inhibited curcumin-induced apoptosis by targeting p21 in Huh7 cells (13), and Jiang et al showed that HCC patients with good survival rates had high miR-345 expression level compared with that in cases with poor survival rates (14). 
Thus, it is worth investigating the biological role of miR-345 and its underlying mechanisms in HCC.

This study showed that miR-345 underexpression was observed in HCC tissues and cells. We also illustrated that loss of miR-345 promoted HCC cell migration and invasion, and resulted in epithelial-mesenchymal-transition (EMT) progression probably by targeting interferon regulatory factor 1 (IRF1)-mediated mTOR/STAT3/AKT signaling in vitro. Thus, this work supported the first evidence that miR-345 was recognized as a potential therapeutic target for HCC.

\section{Materials and methods}

Cell culture and transfection. HCC-derived cell lines (HepG2, SMMC-7721, MHCC97L, MHCC97H and HCCLM3) and a normal hepatocyte cell line (LO2) were purchased from the Cell Bank of Shanghai Institute of Cell Biology, Chinese Academy of Medical Science (Shanghai, China). Cell lines were cultured in DMEM with $10 \%$ fetal bovine serum (Gibco, Grand Island, NY, USA) with anti-biotics (Sigma-Aldrich, St. Louis, MO, USA) at $37^{\circ} \mathrm{C}$ in $5 \% \mathrm{CO}_{2}$.

miR-345 mimic (HmiR0210-MR04), miR-345 inhibitor (HmiR-AN0437-AM04), IRF1 overexpression plasmid (pcDNA3.1-IRF1) and corresponding negative control vectors (CmiR0001-MR04; CmiR-AN0001-AM04) were designed and synthesized by GeneCopoeia (Guangzhou, China). Cell transfection was performed by using Lipofectamine 2000 (Invitrogen, Carlsbad, CA, USA) according to the supplier's protocol.

Quantitative real-time polymerase chain reaction ( $q R T$ $P C R)$. Total RNA was extracted from the cells and tissues with TRIzol reagent (Invitrogen). PrimeScript RT Master Mix Perfect Real-time (Takara, Shiga, Japan) was used to assess IRF1, while Poly-A polymerase based First-Strand Synthesis kit (Takara) was used for miR-345 by polyadenylating the total RNA. After reverse transcription, qRT-PCR was performed by using SYBR Premix Ex Taq II (Takara). IRF1 was normalized to GAPDH, while U6 was used as miR-345 endogenous control. The primers used for miR-345, U6, IRF1 and GAPDH were designed and synthesized by Sangon Biotech (Shanghai, China).

Western blotting. Antibodies for the western blot were as follows: IRF1 (Cell Signaling, Danvers, MA, USA), mTOR (Cell Signaling), p-mTOR (Ser2448; Cell Signaling), STAT3 (Cell Signaling), p-STAT3 (Tyr705; Cell Signaling), AKT (Cell Signaling), p-AKT (Ser473; Cell Signaling), E-cadherin (Abcam, Cambridge, MA, USA), N-cadherin (Abcam), vimentin (Abcam) and GAPDH (G8140, US Biological, Swampscott, MA, USA). The rabbit/mouse secondary antibodies were obtained from Cell Signaling. Two days post transfection, total protein was extracted, following by quantification with BCA protein assay kit (Pierce, Bonn, Germany). Proteins were separated by $10 \%$ SDS-polyacrylamide gels and subsequently transferred to PVDF membranes. After blocking, blots were probed with specific antibodies.

Wound healing assay. HCC cells transduced with corresponding vectors were seeded in 6-well plates to form single
Table I. Clinicopathological correlation of miR-345 expression in HCC.

\begin{tabular}{|c|c|c|c|c|}
\hline \multirow[b]{2}{*}{$\begin{array}{l}\text { Clinicopathological } \\
\text { features }\end{array}$} & \multirow[b]{2}{*}{ Cases } & \multicolumn{2}{|c|}{$\begin{array}{c}\text { miR-345 } \\
\text { expression }\end{array}$} & \multirow[b]{2}{*}{ P-value } \\
\hline & & $\begin{array}{c}\text { Low } \\
(n=33)\end{array}$ & $\begin{array}{l}\text { High } \\
(n=32)\end{array}$ & \\
\hline \multicolumn{4}{|l|}{ Age (y) } & 0.515 \\
\hline$\leq 50$ & 27 & 15 & 12 & \\
\hline$>50$ & 38 & 18 & 20 & \\
\hline \multicolumn{4}{|l|}{ Gender } & 0.518 \\
\hline Male & 49 & 26 & 23 & \\
\hline Female & 16 & 7 & 9 & \\
\hline \multicolumn{4}{|l|}{$\mathrm{HBsAg}$} & 0.158 \\
\hline Absent & 21 & 8 & 13 & \\
\hline Present & 44 & 25 & 19 & \\
\hline \multicolumn{4}{|c|}{ Serum AFP level (ng/ml) } & 0.675 \\
\hline$\leq 20$ & 24 & 13 & 11 & \\
\hline$>20$ & 41 & 20 & 21 & \\
\hline \multicolumn{4}{|l|}{ Tumor size (cm) } & 0.170 \\
\hline$\leq 5$ & 25 & 10 & 15 & \\
\hline$>5$ & 40 & 23 & 17 & \\
\hline \multicolumn{4}{|l|}{ No. of tumor nodules } & $0.019^{\mathrm{a}}$ \\
\hline 1 & 51 & 22 & 29 & \\
\hline$\geq 2$ & 14 & 11 & 3 & \\
\hline \multicolumn{4}{|l|}{ Cirrhosis } & 0.173 \\
\hline Absent & 27 & 11 & 16 & \\
\hline Present & 38 & 22 & 16 & \\
\hline \multicolumn{4}{|l|}{ Venous infiltration } & $0.014^{\mathrm{a}}$ \\
\hline Absent & 48 & 20 & 28 & \\
\hline Present & 17 & 13 & 4 & \\
\hline \multicolumn{4}{|c|}{ Edmondson-Steiner grading } & 0.098 \\
\hline $\mathrm{I}+\mathrm{II}$ & 49 & 22 & 27 & \\
\hline $\mathrm{III}+\mathrm{IV}$ & 16 & 11 & 5 & \\
\hline \multicolumn{4}{|l|}{ TNM tumor stage } & $0.010^{\mathrm{a}}$ \\
\hline $\mathrm{I}+\mathrm{II}$ & 50 & 21 & 29 & \\
\hline III+IV & 15 & 12 & 3 & \\
\hline
\end{tabular}

HCC, hepatocellular carcinoma; HBV, hepatitis B virus; AFP,

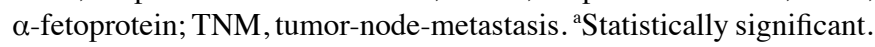

confluent cell layer. The wound were made with $100 \mu \mathrm{l}$ tips in the confluent cell layer. At 0 and $24 \mathrm{~h}$ after would scratching, the width of wound was photographed with a phase-contrast microscope.

Transwell invasion assay. We determined the cell invasion capacities by using Transwell chambers of pore size $8 \mu \mathrm{m}$ (Corning Costar, Cambridge, MA, USA). At $24 \mathrm{~h}$ after transfection, $5 \times 10^{4}$ cells were seeded in the 1:9 diluted Matrigel-coated (BD Biosciences, Franklin Lakes, NJ, USA) 

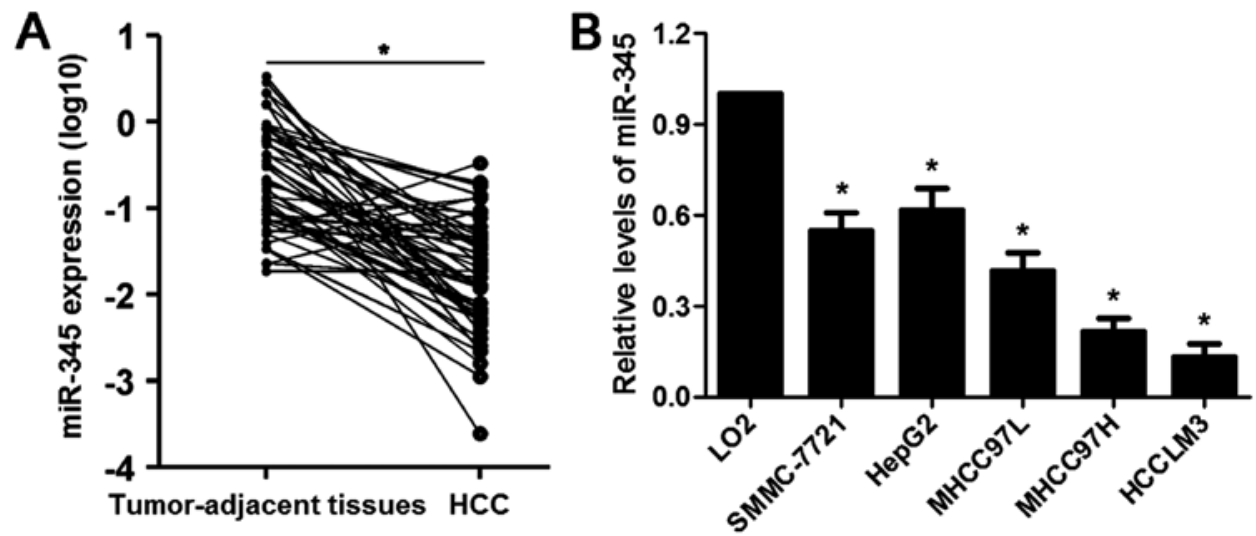

Figure 1. miR-345 is underexpressed in HCC tumor tissues and cells. (A) qRT-PCR was performed to identify the alterative expression of miR-345 in 65 pairs of human HCC and matched adjacent normal liver tissues. "P<0.05 by t-test. (B) The differences of expression of miR-345 between HCC cell lines (SMMC7721, HepG2, MHCC97L, MHCC7H and HCCLM3) and a normal hepatocyte cell line (LO2). Three repeats with similar results, ${ }^{*} \mathrm{P}<0.05$ by ANOVA.

upper chamber with $250 \mu 1$ serum-free DMEM medium, while $700 \mu \mathrm{l}$ DMEM medium with $10 \%$ serum were added in the lower chamber. After $24 \mathrm{~h}$, we fixed cells with paraformaldehyde and the cells in upper chamber were removed. Cells in lower chamber were then stained using $0.1 \%$ crystal violet solution and photographed.

Dual-luciferase reporter assay. Wild-type (wt) or mutant (mt) 3'-UTR of IRF1 was amplified and cloned into pmiRRB-REPORT ${ }^{\mathrm{TM}}$ Luciferase. Luciferase reporter containing the potential binding sequence of 3'-UTR of IRF1 was co-transfected with miR-345 mimic and corresponding negative control vector in HCCLM3 cells in 96-well plate. Two days later, dual-luciferase reporter assay system (Promega, Madison, WI, USA) was used to measure the alteration of luciferase. Firefly luciferase activity was normalized to renilla luciferase activity.

Immunofluorescence (IF). HCC cells were seeded on chamber slides and were fixed with $4 \%$ paraformaldehyde at room temperature (RT) for $10 \mathrm{~min}$. Then, primary antibodies against E-cadherin (Abcam) or vimentin (Abcam) were subjected to cell incubation at $4^{\circ} \mathrm{C}$ overnight. Then, the slides were incubated with matched secondary antibodies (Invitrogen) for $1 \mathrm{~h}$ at RT. The nuclear of HCC cells were stained with DAPI (Sigma) for $10 \mathrm{~min}$ at RT. LSM 5 Pascal Laser Scanning Microscope was used for capturing fluorescence confocal images (Zeiss Germany, Oberkochen, Germany).

Immunohistochemistry (IHC). The tumor tissues that were previously formalin-fixed and paraffin-embedded were sliced into $4 \mu \mathrm{m}$ sections, and underwent deparaffination and then rehydration. Antigen retrieval, suppression of endogenous peroxidase activity and $10 \%$ skim milk blocking were performed before primary antibody incubation. IRF1 (Cell Signaling) antibody was used as primary antibody overnight at $4^{\circ} \mathrm{C}$. The following day, the slides were incubated with peroxidase conjugated secondary antibody (ZSGB-BIO, Beijing, China) for $90 \mathrm{~min}$, and a peroxidase-labeled polymer, DAB solution was used for signal development for $5 \mathrm{~min}$. The sections were counterstained with hematoxylin followed by dehydrating and mounting.
In vivo liver metastasis assay. HCCLM3 cells $\left(6 \times 10^{6}\right)$ that were transfected with miR-345 mimic or control vector were intravenously injected into nude mice. Five weeks after injection, all mice were euthanized, and the livers were sectioned and stained by H\&E to check if hepatic metastatic foci formed. The protocol for these animal experiments were approved by the Ethics Review Committee of Zhengzhou University.

Patients and tissue samples. Sixty-five pairs of human $\mathrm{HCC}$ and matched tumor-adjacent tissues were obtained from HCC patients undergoing hepatectomy at Department of Hepatobiliary Surgery, People's Hospital of Zhengzhou University. All tissue samples were stored in liquid nitrogen or fixed with $10 \%$ formalin after surgical excision immediately. The patients did not receive any anti-tumor therapy before surgical excision. Each patient was well informed and signed the informed consent. The study was approved by the ethics committee of Zhengzhou University in accordance with the Declaration of Helsinki.

Statistical analysis. Data are presented as mean \pm SEM and analyzed by GraphPad Prism5 software (GraphPad Software, Inc., San Diego, CA, USA). Chi-squared test was employed to explore the association between two variables. The Student's t-test and ANOVA were carried out to analyze continuous variable. Correlation curve was constructed and differences among groups were calculated using the Spearman's rank correlation analysis. P-value $<0.05$ was considered to indicate a statistically significant difference.

\section{Results}

Loss of miR-345 promotes invasion and migration of HCC cell lines in vitro. The mRNA expression levels of miR-345 in 65 cases of HCC and matched tumor-adjacent specimens were evaluated by qRT-PCR. Our data illustrated that the expression of miR-345 was significantly downregulated in HCC cases $(\mathrm{P}<0.05$, Fig. 1A). Underexpression of miR-345 was found in HCC cell lines (SMMC-7721, HepG2, MHCC97L, MHCC97H and HCCLM3) compared to LO2 cells $(\mathrm{P}<0.05$, respectively, Fig. 1B). The median of all HCC samples was considered as a cut-off value and the level of miR-345 was 

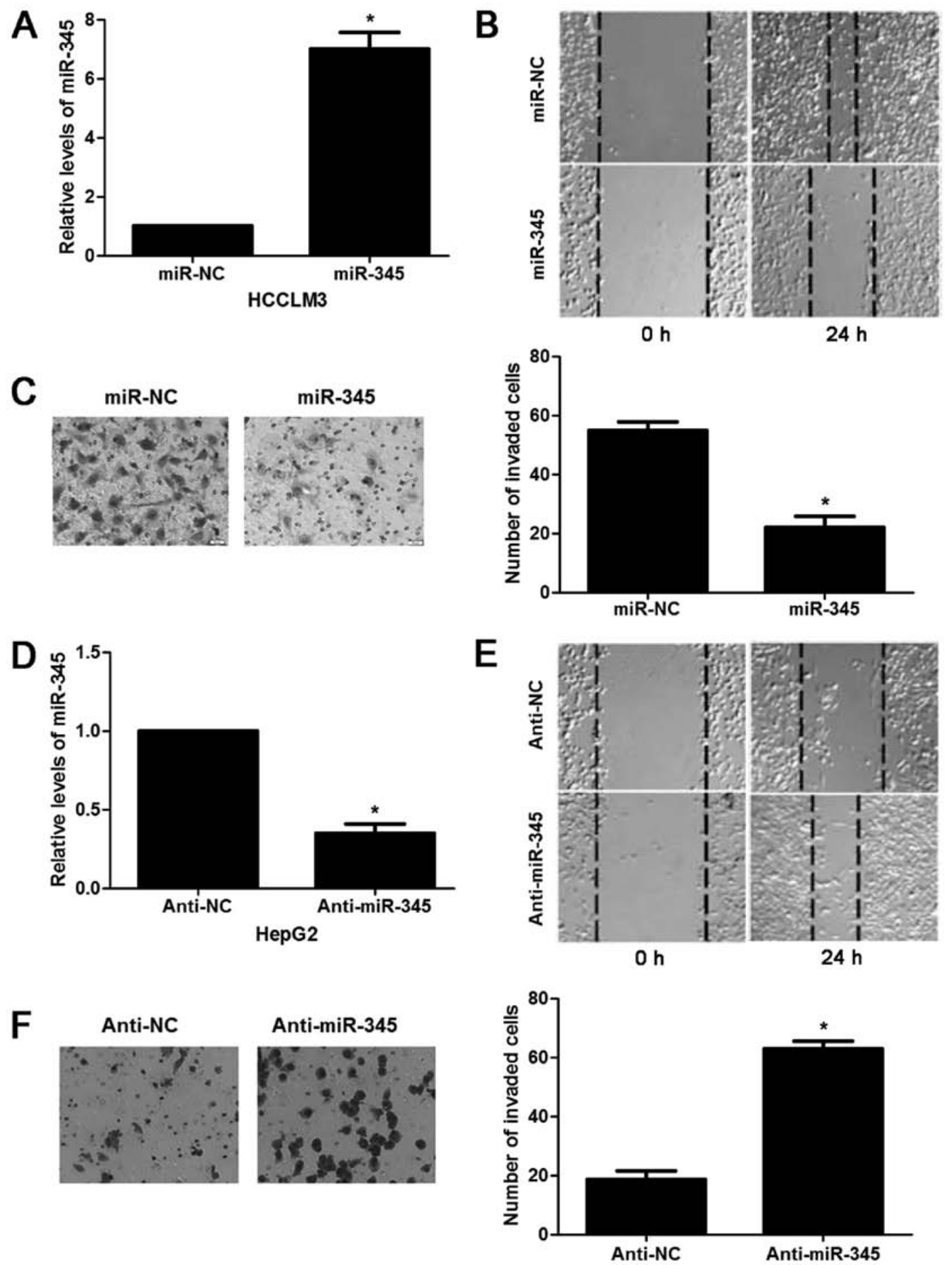

Figure 2. miR-345 regulates cell migration and invasion in HCC cells. (A) HCCLM3 cells that were transduced with miR-345 mimic and corresponding negative control vector (miR-NC) were detected with qRT-PCR for miR-345 expression. Three repeats with similar results, "P<0.05 by t-test. (B) Wound healing assays indicated that miR-345 overexpression suppressed cell migration in HCCLM3 cells. (C) The invasion capacity of HCCLM3 cells were measured by Transwell assays after miR-345 mimic treatments. Three repeats with similar results, ${ }^{*} \mathrm{P}<0.05$ by t-test. (D) HepG2 cells that were transduced with miR-345 inhibitor (anti-miR-345) and corresponding negative control vector (anti-NC) were assessed by qRT-PCR for miR-345 expression. Three repeats with similar results, ${ }^{\prime} \mathrm{P}<0.05$ by t-test. (E) Wound healing assays revealed that miR-345 loss promoted cell migration in HepG2 cells. (F) The invasion capacity of HepG2 cells were enhanced as measured by Transwell assays after miR-345 silencing. $n=3$ repeats with similar results, ${ }^{*} \mathrm{P}<0.05$ by $\mathrm{t}$-test.

divided into low ( $\leq$ median, $\mathrm{n}=33$ ) or high ( $>$ median, $\mathrm{n}=32$ ) group. Clinical correlation analysis disclosed that the low expression of miR-345 was associated with multiple tumor nodes, venous infiltration and advanced tumor-node-metastasis (TNM) stage $(\mathrm{P}<0.05$, respectively, Table I). Next, we tested the effects of miR-345 on invasion and migration capacity of $\mathrm{HCC}$ in vitro. miR-345 mimic resulted in significant increase of miR-345 expression in HCCLM3 cells ( $\mathrm{P}<0.05$, Fig. 2A). miR-345 overexpression inhibited migration and invasion ability of HCCLM3 cells ( $\mathrm{P}<0.05$, respectively, Fig. $2 \mathrm{~B}$ and C). While the opposite results were observed after anti-miR-345 transfection in HepG2 cells $(\mathrm{P}<0.05$, Fig. 2D-F). In the lung metastasis nude mouse model, miR-345 overexpression notably reduced the lung metastases of HCC cells in vivo 


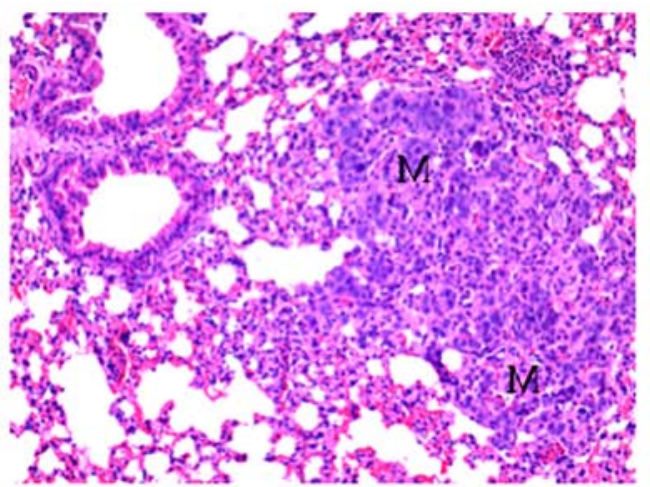

miR-NC
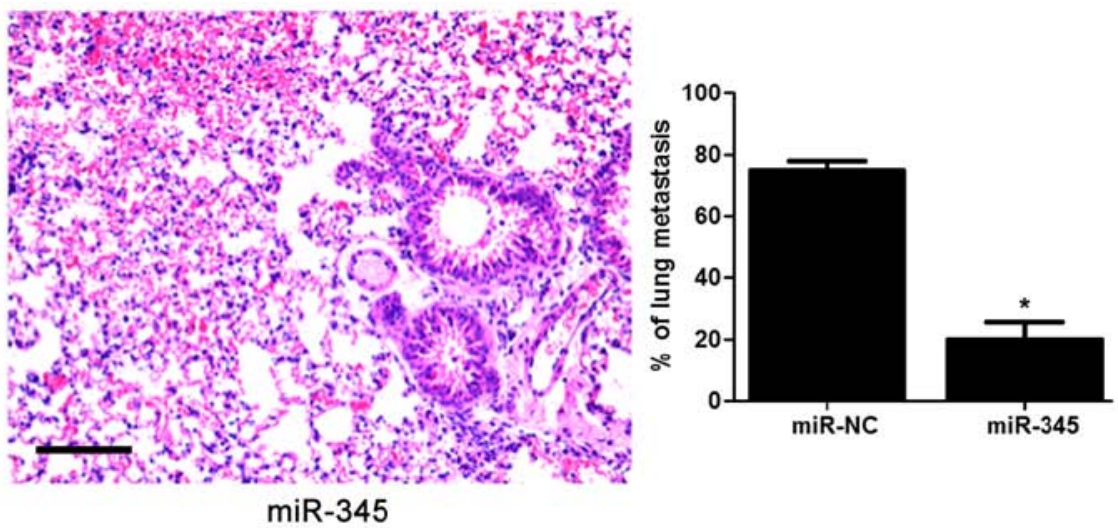

Figure 3. miR-345 overexpression reduced lung metastases in mice. HCCLM3 cells that were transfected with miR-345 mimic and corresponding negative control vector (miR-NC) were intravenously injected into nude mice ( $\mathrm{n}=8$ for each group). H\&E staining revealed that miR-345 overexpression significantly reduced lung metastases of HCCLM3 cells in vivo. ${ }^{*} \mathrm{P}<0.05$ by t-test. M, metastases; scale bar, $50 \mu \mathrm{m}$.

A

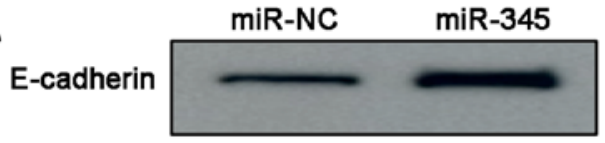

Vimentin

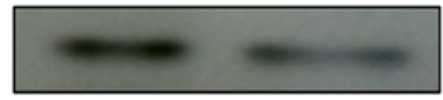

$\mathrm{N}$-cadherin

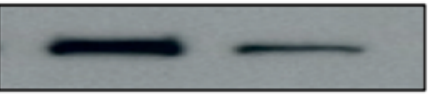

GAPDH
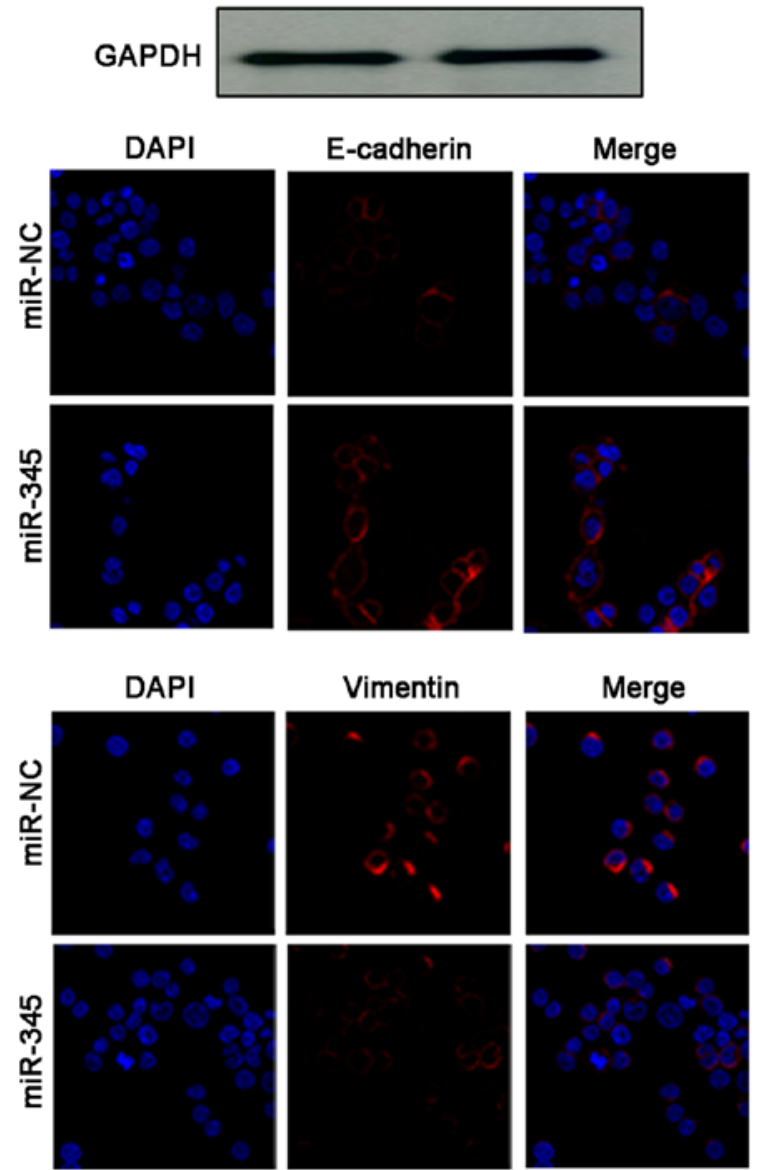

B
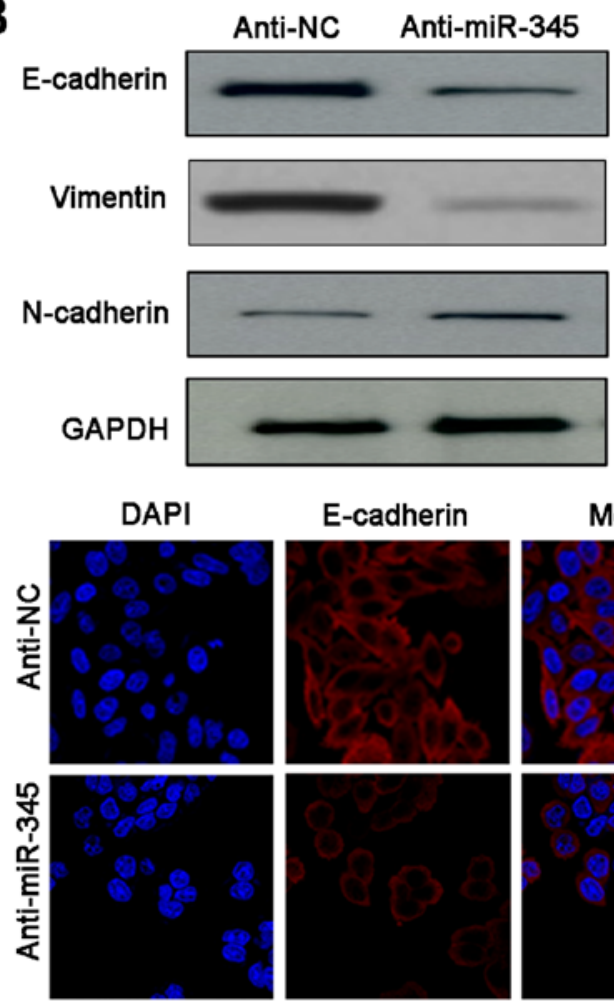

DAPI
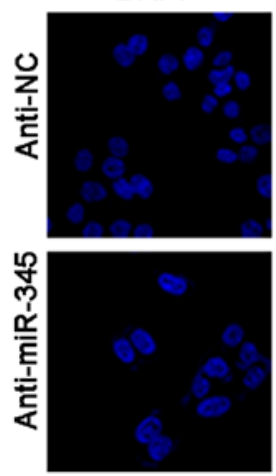
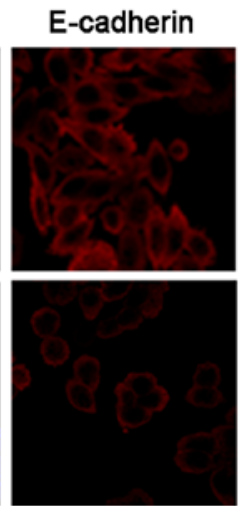

Vimentin
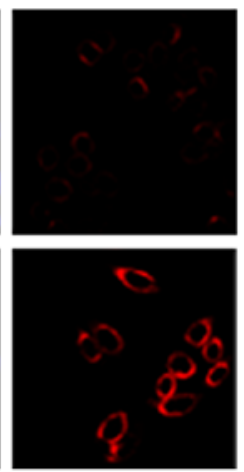

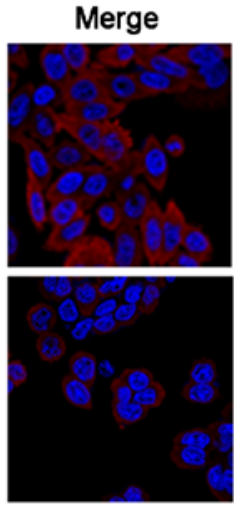

Merge
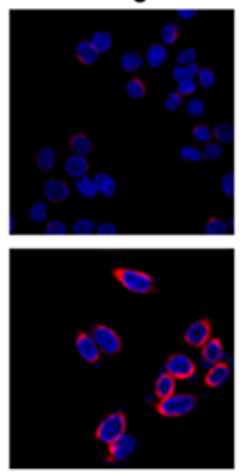

Figure 4. Loss of miR-345 results in enhanced EMT in HCC cell lines. (A) miR-345 mimic treatment upregulated the level of epithelial marker (E-cadherin) and resulted in reduced expression of mesenchymal markers (N-cadherin and vimentin) in HCCLM3 cells. The biomarkers of EMT (E-cadherin and vimentin) in miR-345 overexpressed HCCLM3 cells and control cells were further verified by IF. (B) Downregulation of E-cadherin and upregulation of N-cadherin and vimentin were observed in miR-345 silenced HepG2 cells. Immunostaining of E-cadherin and vimentin were confirmed by IF in anti-miR-345 treated HepG2 cells and control cells. 
A
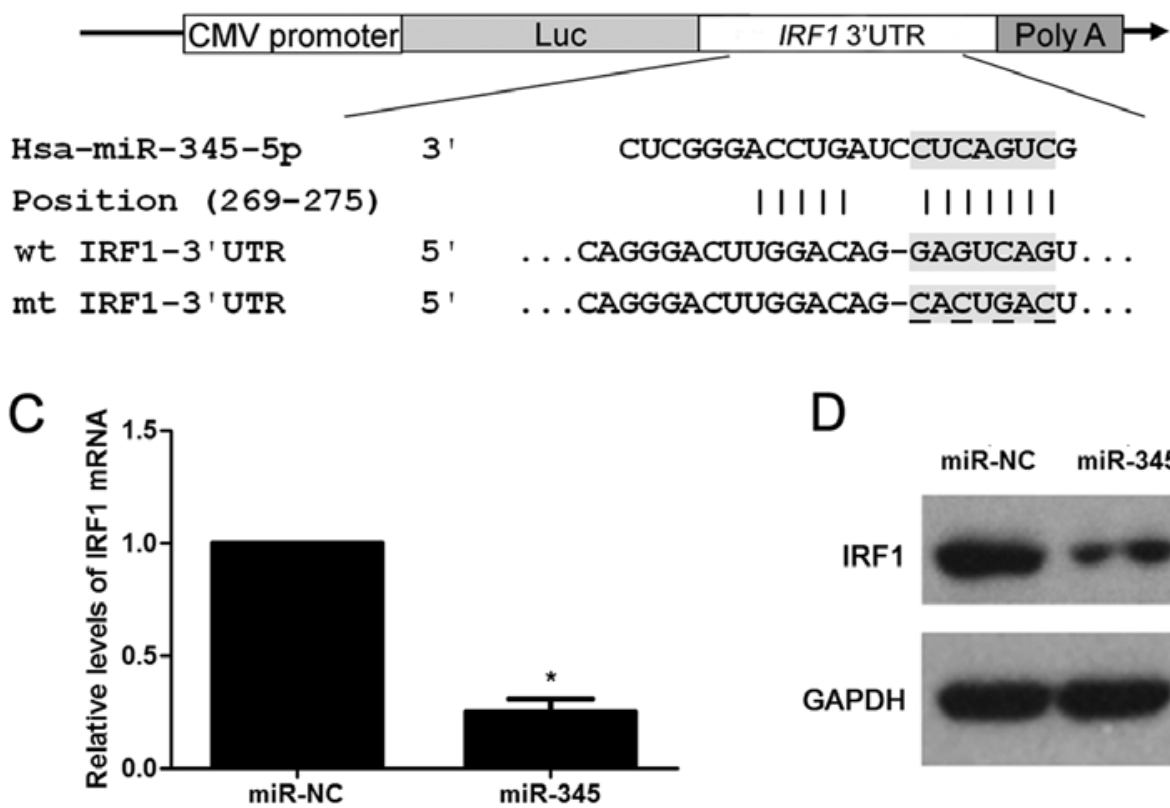
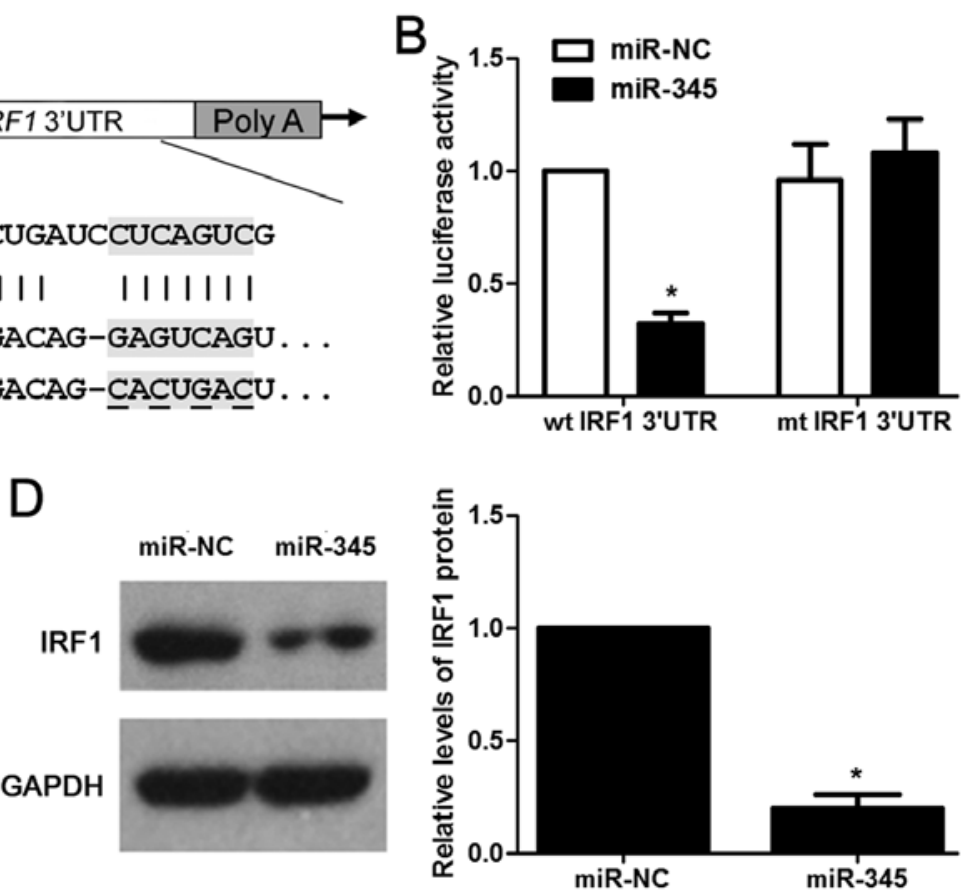

Figure 5. IRF1 is the direct target of miR-345 in HCC cells. (A) The potential miR-345 binding site in wild-type (wt) 3'-UTR sequence of IRF1. The underlined part is the mutant site designed for mutant (mt) 3'-UTR sequence of IRF1. (B) HCCLM3 cells were co-transduced with miR-NC or miR-345 and luciferase reporters containing 3'-UTR of IRF1 sequence. miR-345 overexpression reduced the luciferase activity of wt IRF1 3'-UTR, while showed no significant effect on the luciferase activity mt IRF1 3'-UTR. $\mathrm{n}=3$ repeats with similar results, ${ }^{*} \mathrm{P}<0.05$ by t-test. (C and D) The regulatory role of miR-345 on IRF1 expression in HCC cells was determined by western blot and qRT-PCR analysis. Three repeats with similar results, ${ }^{*} \mathrm{P}<0.05$ by t-test.

( $\mathrm{P}<0.05$, Fig. 3). These data uncovered an anti-invasive role of miR-345 in HCC cell lines.

Underexpression of $m i R-345$ promotes the EMT process of HCC cells. As miR-345 harboured anti-invasive ability in HCC, we then explored the underlying relationship between miR-345 and EMT. Noteworthy, western blotting and IF results indicated remarkable gain of epithelial marker (E-cadherin) and obvious loss of mesenchymal markers ( $\mathrm{N}$-cadherin, vimentin) were detected after miR-345 restoration in HCCLM3 cells (Fig. 4A). Conversely, anti-miR-345 reduced the expression of E-cadherin as well as upregulated the levels of $\mathrm{N}$-cadherin and vimentin in HepG2 cells (Fig. 4B). Therefore, our data demonstrated that loss of miR-345 enhanced invasion and migration abilities of HCC cells by promoting EMT process.

IRF1 is the direct target for miR-345 in HCC. According to the prediction of bioinformatic software (Targetscan, miRanda and PicTar), IRF1 is considered as one of the candidates with which miR-345 could bind directly (Fig. 5A). We then evaluated the ability of miR-345 to inhibit the IRF1 expression via dual-luciferase reporter assay. miR-345 overexpression obviously inhibits luciferase activity of the reporter with wt 3'-UTR sequence of IRF1 ( $\mathrm{P}<0.05$, Fig. 5B), miR-345 restoration showed no significant effect on luciferase activity of $\mathrm{mt}$ 3'UTR of IRF1 (Fig. 5B). We next tested whether miR-345 could participate in the modulation of IRF1. The data of gain-of-function experiments in HCCLM3 cells revealed that the expression of IRF1 could be significantly downregulated by miR-345 overexpression at both the mRNA and protein level ( $\mathrm{P}<0.05$, respectively, Fig. 5C and D). Hence, miR-345 downregulated the expression of IRF1 in HCC cells by binding to its 3'-UTR sequence directly according to the data in context. Furthermore, HCC cases were subjected to qRT-PCR for IRF2 mRNA expression. Quantitative data disclosed that IRF2 mRNA was overexpressed in HCC specimens compared to matched tumor-adjacent tissues $(\mathrm{P}<0.05$, Fig. 6A). The expression levels of miR-345 expression was negatively correlated with the levels of IRF1 mRNA in HCC specimens analyzed by Spearman's rank correlation analysis $(\mathrm{r}=-0.415, \mathrm{P}<0.001$, Fig. 6B). Representative IHC sections showed that miR-345 low expressing HCC showed strong staining of IRF1, while weak signal was detected in miR-345 high expressing cases (Fig. 6C).

Re-expression of IRF1 reverses miR-345-inhibited EMT process in $H C C$. We next detected whether re-expression of IRF1 would rescue the effects of miR-345 in HCC. As expected, restoration of IRF1 rescued the prohibited effect of miR-345 on IRF1, as well as EMT-related biomarkers (E-cadherin, $\mathrm{N}$-cadherin and vimentin) (Fig. 7A). The migration and invasion ability had also been measured and as shown in Fig. 7B and $\mathrm{C}$ : decreased migratory and invasive ability caused by miR-345 was sequentially increased by IRF1 overexpression in HCCLM3 cells $(\mathrm{P}<0.05$, respectively). Hence, these results further confirmed that loss of miR-345 promoted EMT and cell mobility of HCC by targeting IRF1.

mTOR/STAT3/AKT signaling axis may involved in the miR-345/IRF1 mediated EMT. As previous research has identified that IRF1 activated mTOR/STAT3/AKT signaling 

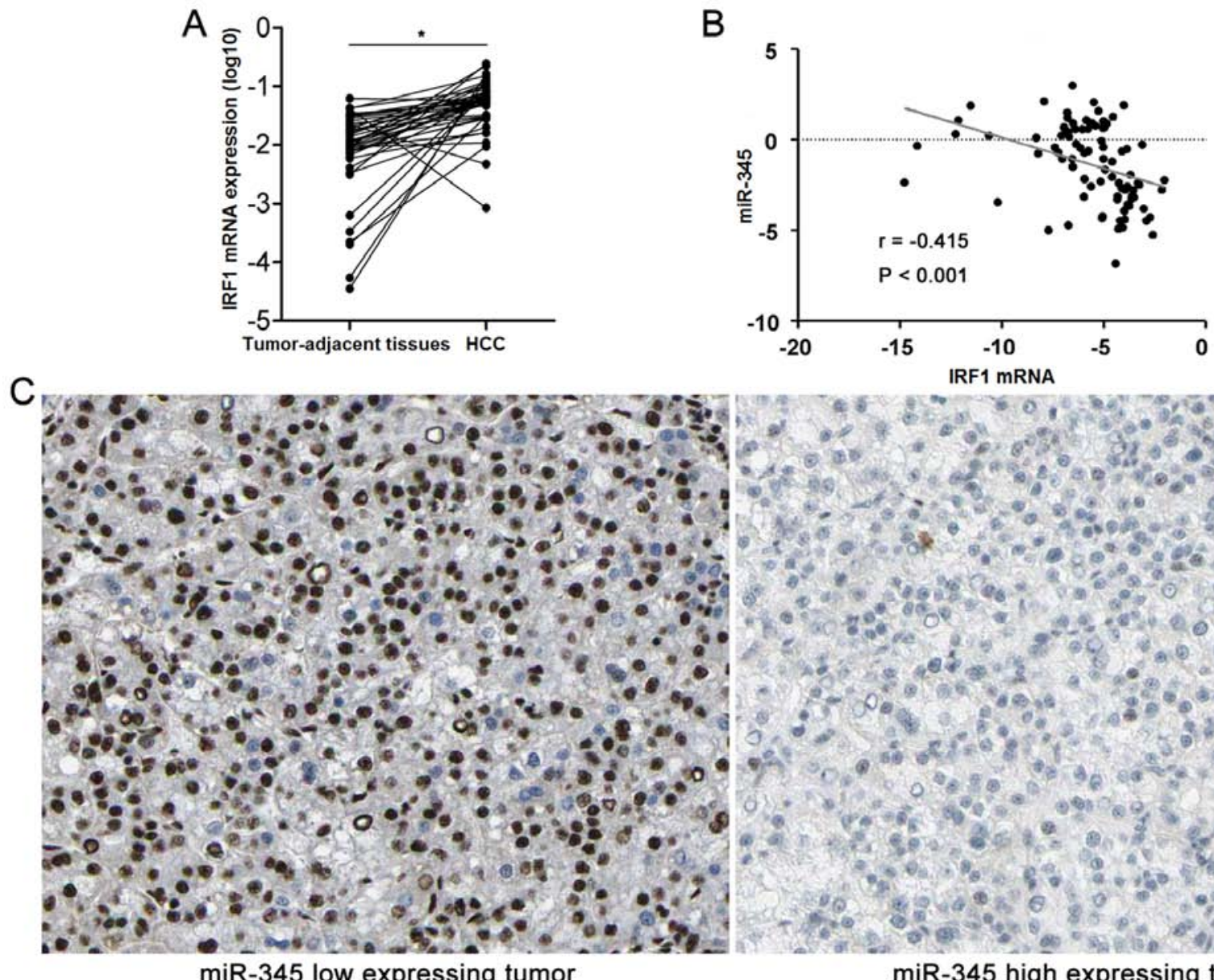

miR-345 low expressing tumor

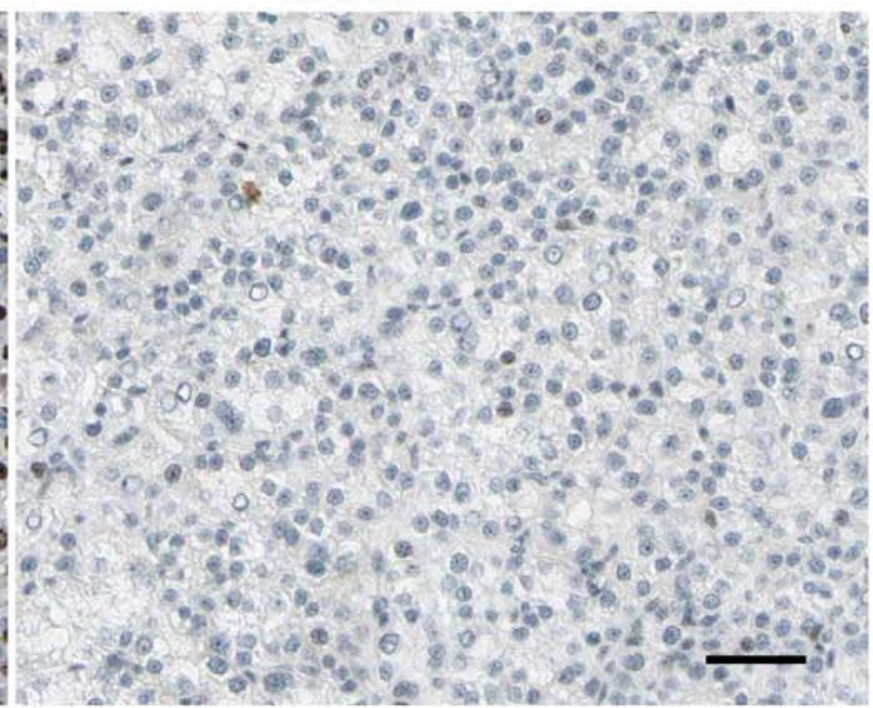

miR-345 high expressing tumor

Figure 6. Negative correlation between miR-345 and IRF1 expression in HCC. (A) qRT-PCR data revealed that the expression levels of IRF1 mRNA were upregulated in HCC compared to matched tumor-adjacent tissues ( $\mathrm{n}=65)$. " $\mathrm{P}<0.05$ by $\mathrm{t}$-test. (B) The expression of miR-345 was negatively correlated with IRF1 mRNA in HCC specimens confirmed by Spearman's rank correlation analysis. $n=65$. (C) Representative IHC sections showed that miR-345 low expressing HCC showed strong staining of IRF1, while weak signal was detected in miR-345 high expressing cases. Scale bar: $50 \mu \mathrm{m}$.

and subsequently upregulated Slug, Snail and Twist, which could promote HCC cell migration, invasion as well as EMT process (15) and we continued to explore whether mTOR/ STAT3/AKT signaling axis is involved in the miR-345/IRF1 mediated EMT process. Of note, we found that the levels of phosphoryated mTOR, STAT3 and AKT as well as Slug, Snail and Twist were remarkably decreased in the miR-345 overexpression group while miR-345 knockdown lead to increased activity of mTOR/STAT3/AKT signaling pathway and its downstream targets (Fig. 8). Therefore, these results indicated that mTOR/STAT3/AKT signaling axis might be involved in the miR-345/IRF1 mediated EMT process in HCC.

\section{Discussion}

Recent research has demonstrated that miRNAs are involved in tumor initiation and progression as either oncogenes or tumor suppressors by negatively regulating downstream targets (16-18). Therefore, identifying the potential valuable miRNAs provides novel insight for the diagnosis and treatment of patients with HCC $(3,16)$. Herein, our data indicated that underexpressed miR-345 was common in HCC tissues. In addition, the levels of miR-345 were intensively reduced in HCC cell lines compared to a normal hepatocyte cell line
(LO2). HCC patients with multiple tumor nodes, venous infiltration and advanced TNM stage showed significantly higher levels of miR-345, which was consistent with poor prognosis prediction as reported by Jiang et al (14). Although great efforts have been put into the study of miR-345 on tumor cell growth, apoptosis and metastasis (7,8,10-13), the roles of miR-345 in the modulation of EMT process remain largely unelucidated in HCC. In our recent study, gain- and loss-of-function methods were used to determine the functional roles of miR-345 in EMT. After restoration of miR-345, upregulated E-cadherin (epithelial marker), suppressed $\mathrm{N}$-cadherin and vimentin (mesenchymal marker) were detected, accompanying with inhibited cell invasion and migration ability in HCCLM3 cells. Contrary data were obtained with anti-miR-345 treatment in HepG2 cells. These results demonstrated that miR-345 served as an tumor suppressor by suppressing EMT in HCC.

The role of IRF1 in HCC is still controversial according to previous studies. Kröger et al reported that the tumor growth of HCC was inhibited by the means of enhanced antitumor growth effect and immune cell recognization (19). Furthermore, IRF1 signaling pathway was implicated in interferon-gamma (IFN- $\gamma$ ) induced autophagy in Huh7 cells (20). While, recent study indicated that suppression of 

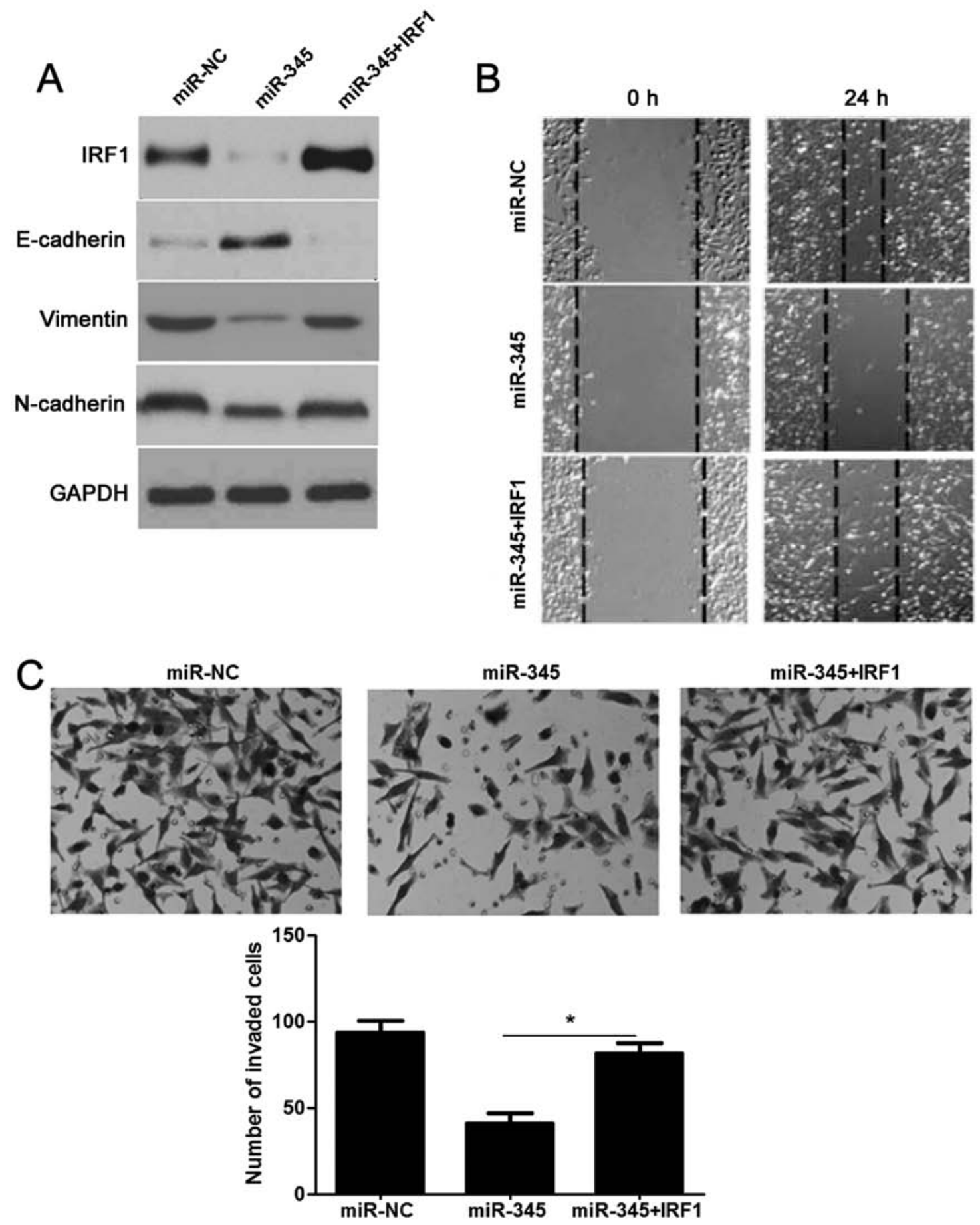

Figure 7. Restoration of IRF1 reversed miR-345 inhibited EMT process in HCC. (A) The expression levels of IRF1 and EMT biomarkers in miR-345 mimic transfected HCCLM3 cells were determined by performing western blotting after IRF1 overexpression. (B) IRF1 restoration facilitated the migration of miR345 overexpressing HCCLM3 cells. (C) Forced expression of IRF1 reversed the inhibitory effects of miR-345 on invasion capacity of HCCLM3 cells. Three repeats with similar results, ${ }^{\mathrm{P}}<0.05$ by ANOVA.

IRF1 was involved in miR-130b induced inhibition of HCC cell migration and invasion (15). In this study, we found IRF1 is a direct target of miR-345, re-expression of IRF1 rescued the miR-345 inhibited EMT process, these results demonstrated miR-345 could inhibit the EMT process by targeting IRF1. Moriyama et al reported that underexpression levels of IRF1 mRNA were observed in 15 of 32 HCC cases compared to matched tumor-adjacent tissues (21). While our data revealed that IRF1 mRNA was overexpressed in HCC specimens. We suggest that the case number and etiology may be the causes of difference. $\mathrm{HCV}$ core protein upregulated the expression of miR-345 in HCC cells (13), while HBV infection is the main problem in China (22).

A previous study showed that IRF1 functions as an oncogenic factor that upregulated p-mTOR, p-STAT3 and p-AKT as well as regulators of EMT including Slug, Snail and Twist, which facilitate HCC cell migration and invasion (15). Hence, we then investigated the effect of IRF1 restoration on the mTOR/STAT3/AKT signaling pathway in order to elucidate the potential mechanism of IRF1 mediated EMT. As expected, 


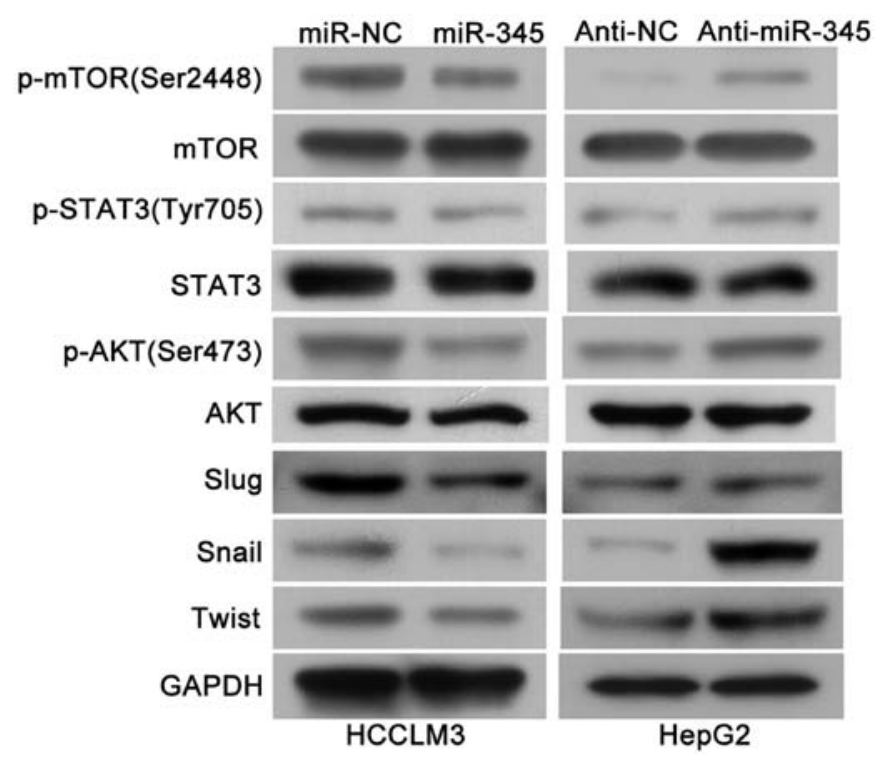

Figure 8. mTOR/STAT3/AKT signaling axis is involved in the miR-345/IRF1 mediated EMT. The activity of mTOR/STAT3/AKT pathway was suppressed by miR-345 mimic treatment with downregulation of p-mTOR, p-STAT3 and p-AKT as well as Slug, Snail and Twist as examined by western blot analysis in HCCLM3 cells. While, miR-345 knockdown enhanced the activity of mTOR/STAT3/AKT pathway in HepG2 cells.

phosphorated AKT, STAT3 and AKT as well as EMT regulators including Slug, Snail and Twist were downregulated in miR-345 overexpressing cells and they were upregulated in the miR-345 knockdown group. These results indicated that mTOR/STAT3/AKT signaling pathway may be involved in the miR-345/IRF1 mediated EMT process. However, further investigations are needed to make a firm conclusion.

To conclude, the underexpression of miR-345 creates a milieu of EMT facilitation that plays a role in HCC progression. A mechanism by which underexpressed miR-345 promotes the EMT by targeting IRF1 plays an important role in this process. This finding will improve understanding of EMT progression mechanism and provide novel targets for the molecular treatment of HCC.

\section{Acknowledgements}

This study was supported by grants from the National Natural Science Foundation of China (no. 81302121).

\section{References}

1. Forner A, Llovet JM and Bruix J: Hepatocellular carcinoma. Lancet 379: 1245-1255, 2012

2. Bruix J and Sherman M; American Association for the Study of Liver Diseases: Management of hepatocellular carcinoma: An update. Hepatology 53: 1020-1022, 2011.

3. Calin GA and Croce CM: MicroRNA signatures in human cancers. Nat Rev Cancer 6, 857-866, 2006.

4. Garzon R, Calin GA and Croce CM: MicroRNAs in cancer. Annu Rev Med 60: 167-179, 2009.
5. Guled M, Lahti L, Lindholm PM, Salmenkivi K, Bagwan I, Nicholson AG and Knuutila S: CDKN2A, NF2, and JUN are dysregulated among other genes by miRNAs in malignant mesothelioma - A miRNA microarray analysis. Genes Chromosomes Cancer 48: 615-623, 2009.

6. Cervigne NK, Reis PP, Machado J, Sadikovic B, Bradley G, Galloni NN, Pintilie M, Jurisica I, Perez-Ordonez B, Gilbert R, et al: Identification of a microRNA signature associated with progression of leukoplakia to oral carcinoma. Hum Mol Genet 18: 4818-4829, 2009.

7. Pogribny IP, Filkowski JN, Tryndyak VP, Golubov A, Shpyleva SI and Kovalchuk O: Alterations of microRNAs and their targets are associated with acquired resistance of MCF-7 breast cancer cells to cisplatin. Int J Cancer 127: 1785-1794, 2010.

8. Tang JT, Wang JL, Du W, Hong J, Zhao SL, Wang YC, Xiong H, Chen HM and Fang JY: MicroRNA 345, a methylation-sensitive microRNA is involved in cell proliferation and invasion in human colorectal cancer. Carcinogenesis 32: 1207-1215, 2011.

9. Schou JV, Rossi S, Jensen BV, Nielsen DL, Pfeiffer P, Høgdall E, Yilmaz M, Tejpar S, Delorenzi M, Kruhøffer M, et al: miR-345 in metastatic colorectal cancer: A non-invasive biomarker for clinical outcome in non-KRAS mutant patients treated with 3rd line cetuximab and irinotecan. PLoS One 9: e99886, 2014.

10. Chen L, Li X and Chen X: Prognostic significance of tissue miR-345 downregulation in non-small cell lung cancer. Int J Clin Exp Med 8: 20971-20976, 2015.

11. Srivastava SK, Bhardwaj A, Arora S, Tyagi N, Singh S, Andrews J, McClellan S, Wang B and Singh AP: MicroRNA-345 induces apoptosis in pancreatic cancer cells through potentiation of caspase-dependent and -independent pathways. Br J Cancer 113: 660-668, 2015.

12. Chen QG, Zhou W, Han T, Du SQ, Li ZH, Zhang Z, Shan GY and Kong CZ: MiR-345 suppresses proliferation, migration and invasion by targeting Smad1 in human prostate cancer. J Cancer Res Clin Oncol 142: 213-224, 2016.

13. Shiu TY, Huang SM, Shih YL, Chu HC, Chang WK and Hsieh TY: Hepatitis C virus core protein down-regulates p21(Waf1/Cip1) and inhibits curcumin-induced apoptosis through microRNA-345 targeting in human hepatoma cells. PLoS One 8: e61089, 2013.

14. Jiang J, Gusev Y, Aderca I, Mettler TA, Nagorney DM, Brackett DJ, Roberts LR and Schmittgen TD: Association of MicroRNA expression in hepatocellular carcinomas with hepatitis infection, cirrhosis, and patient survival. Clin Cancer Res 14: 419-427, 2008.

15. Lin YH, Wu MH, Liao CJ, Huang YH, Chi HC, Wu SM, Chen CY, Tseng YH, Tsai CY, Chung IH, et al: Repression of microRNA-130b by thyroid hormone enhances cell motility. J Hepatol 62: 1328-1340, 2015.

16. Zhu Z, Zhang X, Wang $\mathrm{G}$ and Zheng $\mathrm{H}$ : Role of MicroRNAs in hepatocellular carcinoma. Hepat Mon 14: e18672, 2014.

17. Yao Y, Suo AL, Li ZF, Liu LY, Tian T, Ni L, Zhang WG, Nan KJ, Song TS and Huang C: MicroRNA profiling of human gastric cancer. Mol Med Rep 2: 963-970, 2009.

18. Jansson MD and Lund AH: MicroRNA and cancer. Mol Oncol 6: 590-610, 2012.

19. Kröger A, Ortmann D, Krohne TU, Mohr L, Blum HE, Hauser H and Geissler M: Growth suppression of the hepatocellular carcinoma cell line Hepa1-6 by an activatable interferon regulatory factor-1 in mice. Cancer Res 61: 2609-2617, 2001.

20. Li P, Du Q, Cao Z, Guo Z, Evankovich J, Yan W, Chang Y, Shao L, Stolz DB, Tsung A, et al: Interferon- $\gamma$ induces autophagy with growth inhibition and cell death in human hepatocellular carcinoma (HCC) cells through interferon-regulatory factor-1 (IRF-1). Cancer Lett 314: 213-222, 2012.

21. Moriyama Y, Nishiguchi S, Tamori A, Koh N, Yano Y, Kubo S, Hirohashi K and Otani S: Tumor-suppressor effect of interferon regulatory factor-1 in human hepatocellular carcinoma. Clin Cancer Res 7: 1293-1298, 2001.

22. Tanaka M, Katayama F, Kato H, Tanaka H, Wang J, Qiao YL and Inoue M: Hepatitis B and C virus infection and hepatocellular carcinoma in China: A review of epidemiology and control measures. J Epidemiol 21: 401-416, 2011. 